\title{
Sistemas Agroindustriais Integrados Uma Análise por meio da Entropia de Informação
}

\section{Marcos Ximenes Ponte}

Professor do Núcleo de Altos Estudos Amazônicos e do Departamento de Engenharia Mecânica da Universidade Federal do Pará

\section{Resumo}

Utilizando a teoria matemática da informação, este artigo analisa quatro sistemas agroindustriais de produção de bioenergia: a indústria de álcool de cana-de-açúcar do Brasil, a indústria de álcool de milho e a indústria de biodiesel dos Estados Unidos, além da indústria de farinha de mandioca da Amazônia. Com base em um importante conjunto de informações empíricas, são feitas avaliações de eficiência energética para diferentes níveis de organização dos sistemas.

\section{Palavras-chave}

Amazônia - Agricultura - Entropia Agroindústria - Bioenergia

\section{Donald L. Van Dyne}

Professor do Departamento de Economia Agrícola da Universidade de Missouri, USA

\section{Abstract}

Using the mathematical theory of the information, this article analyzes four agroindustry systems of bioenergy production: the industry of alcohol from sugar-cane in Brazil, the industry of corn alcohol and the industry of biodiesel in United States, besides the industry of manioc flour from the Amazonian. Based on an important group of empiric information, evaluations of energy efficiency for different organization levels of the systems are made.

\section{Keywords}

Amazon - Agriculture - Entropy Agroindustry - Bioenergy 
Marcos Ximenes Ponte, Donald L. Van Dyne

\section{Introdução}

A teoria da informação oferece uma ferramenta matemática que pode ser aplicada a diversos fenômenos naturais, com várias finalidades. Na realidade, a teoria da informação é capaz de expressar a interlocução que existe entre sistemas ou entre partes identificáveis de um mesmo sistema, expressando, dessa forma, os níveis de organização ou o grau de estruturação dos mesmos. $\mathrm{Na}$ última década, houve um crescente interesse por estudos de eventos complexos, fora do equilíbrio, que os métodos analíticos tradicionais não conseguem formular. Assim, a busca de métodos que permitam uma visão fenomenológica e holística de tais eventos tornou-se uma tarefa muito importante, para a qual a teoria da informação tem contribuído substancialmente.

Em particular, a hipótese de que a descrição fenomenológica de tais sistemas longe do equilíbrio poderia ser realizada por meio da idealização de uma rede de interações com trocas de massa e energia é um importante achado, por propiciar um alargamento do escopo da termodinâmica além dos seus tradicionais limites. Como afirmou Ulanowicz (1986) em sua importante contribuição sobre a fenomenologia dos ecossistemas:

"Thermodynamics is an effort to codify what is common to empirical observation. The power of thermodynamics lies in its generalities,

but this can be achieved only at the expense of deliberately ignoring lower level details".

0 principal benefício desse método é o fato de permitir uma formulação matemática no nível do sistema como um todo, por meio da definição de certos parâmetros que expressam o efeito das múltiplas interações no sistema.

Esse método também se harmoniza com a idéia da Ecologia Industrial, cujo intento é fazer um sistema industrial semelhante aos sistemas naturais (J ackson, 1997). Admite-se a idéia de que a estabilidade dos sistemas naturais é baseada na capacidade para criar uma rede de ligações, de tal forma que os resíduos de uma espécie tornam-se o substrato para outra. A importância da identificação de ligações dentro de e entre setores nas atividades agroindustriais para fazer-se uso máximo de energia e informação contida em cada subproduto é defendida por Tiezzi, Marchettini e Ulgiati (1991). Devido à importância da indústria de biocombustíveis, vários estudos importantes têm sido realizados nesse setor envolvendo Life Cycle Inventory, tais como USDA, USDE (1998), sobre biodiesel, Shapouri, Duffield, Graboski (1995), sobre etanol de milho, e Macedo, Horta (1996), sobre a indústria de etanol no Brasil. Alguns estudos têm igualmente enfatizado a oportunidade de aumentar a eficiência e, conseqüentemente, o valor global de produtos e co-produtos dos sistemas agroindustriais destinados à produção de biocombustíveis. Uma boa solução para isso tem sido obtida pela integração entre as atividades agrícolas e industriais com uma unidade de processamento de resíduos que produz mais energia e/

48 
ou produtos químicos de alto valor (ver Van Dyne, Kaylen, Blasé, 1998; ver também Ponte, Van Dyne, 1998). Algumas importantes conquistas tecnológicas no campo do uso industrial da biomassa estão criando novas opções para a atividade rural. Estão emergindo alguns métodos sofisticados para avaliar como esses recursos podem ser colhidos e transformados em bens úteis, muitas vezes fundamentais para viabilizar a atividade produtiva principal. Uma importante questão, sempre presente, diz respeito à avaliação da eficiência desses sistemas integrados, à comparação entre os mesmos e à melhoria de sua eficiência pelos arranjos integrativos.

A teoria da informação, suplementada com informações subsidiárias sobre os sistemas específicos, tem sido utilizada com sucesso em vários campos de estudos, tais como a Ecologia, a Economia, a Geografia e a Literatura. Svirezhev, Brovkin e Denisenko (1995) aplicaram essa formulação à análise da eficiência de agrossistemas (ver também Denisenko, Svirezhev, Brovkin, 1991).

Neste trabalho estamos complementando os estudos de Svirezhev, Brovkin e Denisenko com novas definições e análises, para compararmos quatro sistemas agroindustriais de produção de bioenergia: a indústria de álcool de cana-de-açúcar do Brasil, a indústria de álcool de milho dos USA, a indústria de biodiesel dos USA e a indústria de farinha de mandioca da Amazônia.

\section{Informações Básicas sobre os Sistemas}

Durante as duas últimas décadas, um significativo esforço foi realizado no sentido de ampliar o uso comercial de biocombustível. O Brasil e os Estados Unidos da América são os países que têm demonstrado maior empenho, com resultados práticos mais expressivos. 0 etanol produzido a partir da cana-deaçúcar ou do milho é o mais importante biocombustível líquido. A produção mundial de etanol, de todas os fontes e para as diversas finalidades (industrial, bebidas, etc. ), foi estimada por Berg (1997) em 31.3 bilhões de litros, em 1996, dos quais $67 \%$ foram utilizados para combustível, $20 \%$ foram aplicados a fins industriais e $13 \%$, usados para bebidas. O Brasil produziu nesse ano 14.5 biIhões de litros (46.3\%) a partir da cana-de-açúcar. Os Estados Unidos produziram 5.7 bilhões de litros (18.2\%), largamente baseados em milho. Portanto as Américas foram os maiores produtores, com um total de 65\%; a Ásia produziu 5.6 bilhões de litros (17.9\%); a Europa, 4.6 bilhões de litros (14.7\%); a África, 0.6 bilhões de litros (1.9\%); a Oceania, 0.2 bilhões de litros $(0,6 \%)$. A cana-deaçúcar é a mais importante matéria-prima utilizada na produção de etanol, especialmente na América do Sul, na América Central e na Ásia.

Biodiesel é um substituto renovável do óleo diesel. Diferentemente do etanol, ainda não é significativamente usado. Na Europa, o biodiesel é utilizado, mas ainda em pequena escala, na sua forma pura ( $100 \%$ biodiesel) ou misturado com diesel de petróleo. Nos Estados Unidos, as especulações e os 
Marcos Ximenes Ponte, Donald L. Van Dyne

estudos sobre a produção de biodiesel têm focalizado a possibilidade de usar óleo de soja como matéria-prima, principalmente porque os Estados Unidos são o maior produtor de óleo de soja do mundo (USDA, USDE, 1998).

A produção de farinha de mandioca não é tipicamente um sistema agroindustrial de produção de bioenergéticos. É, sem dúvida, um importante sistema de produção de alimento em regiões tropicais e é o mais importante suporte alimentar do agricultor de subsistência no Norte e no Nordeste do Brasil. Com muita freqüência, a cultura da mandioca é praticada por meio de agricultura itinerante (shifting cultivation), que é um sistema agrícola no qual uma área desmatada é usada para plantio de culturas temporárias, por poucos anos, e, em seguida, a área é posta em pousio, para que a capoeira, resultante do abandono da área, se restaure. 0 período de rotação é maior do que aquele utilizado na produção agrícola (NRC-CSAEHT, 1993). Esse sistema, que nós denominaremos sistema camponês (peasant system), é muito importante pela sua significância social em regiões dos trópicos úmidos. Também é muito interessante para uma análise comparativa de sustentabilidade, visto que esse sistema usa um processo natural de reconstituição da fertilidade do solo, por meio da rotação de áreas (plantation-fallow), diferentemente dos outros sistemas estudados, que usam energia artificial. Para que seja possível a rotação de cultura no sistema camponês, a área necessária é maior do que a área plantada. É fácil verificar que a razão entre a área necessária $A_{n}$ e a área plantada $A_{p}$ pode ser expressa por

$$
a_{r}=A_{n} / A_{p}=1+t_{r} / t_{p}
$$

onde $t_{r}$ é $o$ tempo (anos) de rotação e $t_{p^{\prime}}$ o tempo (anos) em que é possível plantar numa mesma área. Por exemplo, no Nordeste do Pará (Brasil), o tempo de rotação é de 15 anos, e a mandioca pode ser plantada num mesmo local por 3 anos. Assim, a área necessária seria 6 (seis) vezes maior do que a área plantada. Em relação à produção de mandioca, é ainda importante destacar que existe uma produção agrícola não camponesa (no peasant) desse tubérculo, que usa energia artificial (fertilizantes e mecanização) na produção agrícola.

Os sistemas agroindustriais estudados e suas respectivas fontes de informação são: a indústria canavieira para a produção de etanol de São Paulo, no Brasil (Macedo, Horta, 1996); a indústria americana de etanol baseada em milho (Shapouri, Duffield, Graboski, 1995); a indústria americana de biodiesel baseada em soja (USDA, USDE, 1998); a produção de farinha de mandioca no Estado do Pará (Conto et al., 1997; Ximenes, 1998).

Os cálculos da energia obtida dos resíduos seguiram a metodologia contida em Ponte, Van Dyne (1998) e foram complementados com dados do National Renewable Energy Laboratory-NREL (1997), para milho e soja, e de Macedo e Horta (1996), para cana de açúcar.

Se nós considerarmos a informação contida em NREL $(1994,4)$, segundo a qual os fatores de produção de resíduos para milho e soja são 1.5lb/lb e

50 
uma a duas toneladas de resíduos por acre é mais do que suficiente para proteger a fertilidade do solo e prevenir erosão, é possível demonstrar que pelo menos $57 \%$ das 4.66 toneladas de resíduos produzidos por acre de milho são disponíveis. Por esse critério, é também possível constatar que a colheita de soja produz somente 1.47 toneladas de resíduos por acre. Como visto, é a quantidade média de resíduos requerida para manter a produtividade do solo. Adicionalmente, tem sido afirmado que os resíduos de soja não são apropriados para uso energético comercial em virtude da limitação dos equipamentos de colheita. Por outro lado, os resíduos de soja têm o melhor valor como fertilizante entre os grãos, especialmente pelo seu conteúdo de nitrogênio. 0 valor energético equivalente do fertilizante dos resíduos de soja é de $1777 \mathrm{~kJ} /$ $\mathrm{kg}$ de grãos colhidos, enquanto esse valor para o milho é de $834.7 \mathrm{~kJ} / \mathrm{kg}$.

\section{Formulação do Problema}

\section{Definição do Sistema}

O problema será formulado considerando-se o sistema uma rede ponderada (weighted) de trocas de massa e energia entre seus compartimentos e entre estes e o meio. Uma rede é uma coleção de elementos de um sistema chamados nodos. Esses nodos são juntados um ao outro por elementos chamados edges. Um edge é chamado arco quando é indicado pela ordem em que os nodos estão. Uma seqüência de arcos é chamada de via (pathway). Uma rede pode ser cíclica ou acíclica se possui ou não, uma via cíclica. Se magnitudes são associadas a cada arco, a rede é dita ponderada.

0 sistema industrial bioenergético (SIB), na forma tratada neste trabaIho, é uma rede na qual os nodos são: a unidade agrícola (1), a unidade industrial (2) e a unidade de processamento de resíduos (3) para produzir mais energia e novos produtos. Os nodos podem trocar massa e energia entre si e, externamente, com o meio. Para descrever o SIB em termos de fluxos de massa e energia, é necessário categorizar os fluxos mais significantes e determinar os equivalentes energéticos desses fluxos. Uma vista esquemática dessa rede é mostrada na Figura 1.

Figura 1 - Rede de fluxos entre os compartimentos do sistema agroindustrial.

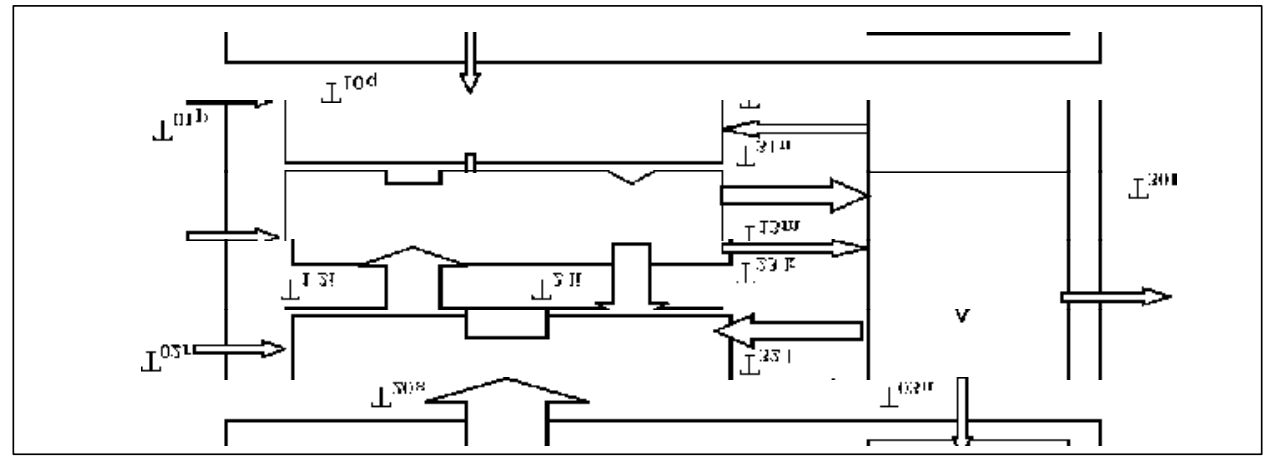


Em uma visão ampla, as seguintes variáveis expressam o comportamento do sistema:
F [condições geográficas ${ }^{(1)}$, padrão tecnológico, tamanho do sistema, grau de integração,conteúdo energético dos materiais circulantes, tempos de produção]

O nosso principal objetivo é expressar o comportamento desse sistema por meio de uma formulação baseada na teoria da informação.

$\mathrm{T}_{\mathrm{ijk}}$ representa um fluxo que se origina no i-gésimo compartimento para j-ésimo compartimento. " $k$ " indica a categoria do fluxo (i.e., se é combustível, fertilizante, construção, maquinaria, trabalho, etc.). "0" denota a posição fora do sistema, por exemplo, $\mathrm{T}_{01 \mathrm{p}}$ é um fluxo de entrada e $\mathrm{T}_{10 \mathrm{q}}$ denota um fluxo de saída no compartimento 1. Quando nós tratamos com todos os tipos de fluxos indistintamente, usa-se a notação $T_{\text {iij, }}$

Ulanowicz $(1986,36)$ define a carga total (throughput) do sistema, em termos de fluxo, como a soma de todos os fluxos no sistema. Esse parâmetro mede o tamanho do sistema inteiro de $\mathrm{n}$ componentes.

$$
T=\sum_{j=1}^{n} \sum_{i=1}^{n} i_{j}
$$

\section{Elementos da Teoria da Informação Aplicados à Análise de um SIB}

Visto que o nosso propósito básico é promover a descrição fenomenológica da estrutura do sistema, por meio de fluxos de matéria e energia, 0 primeiro passo é estabelecer a relação entre esses fluxos em cada compartimento. Se o sistema consiste em n compartimentos, em regime permanente, para cada nodo i nós podemos escrever um balanço de massa e energia, o que resulta em:

$$
T_{0 i}-T_{i 0}+\sum_{j=1 j i}^{n} T_{j=1}^{n} T_{i k}=d E / d t=0
$$

$\mathrm{T}_{0 \mathrm{i}}$ e $\mathrm{Ti}_{0}$ são, respectivamente, o input e o output no compartimento $\mathrm{i}$, as medidas de sua relação com o exterior. A eficiência do sistema é

$$
\eta=\sum_{i=1}^{n} \bar{i}_{i=1}^{n} / \sum b_{i}
$$

É importante notar que a energia solar é uma propriedade do sistema e, portanto, não está incluída na definição de eficiência como um fluxo de entrada. Por isso, em geral a eficiência excede a unidade para sistemas agrobioenergéticos.

As transformações acorrem em um SIB como uma série de transferências discretas, as quais podem assumir diferentes e imprevisíveis valores. A observação sobre a rede de fluxos reduz a incerteza total do sistema. Assim, o conceito de entropia como medida de incerteza de uma vari-

\footnotetext{
${ }^{1}$ Incluem a energia do sol e a fertilidade natural do solo.
}

52 
ável aleatória é um importante instrumento matemático. Shannon (1948) definiu entropia $H(X)$ como uma medida da incerteza da distribuição de probabilidade $p\left(x_{i}\right)$ para uma variável aleatória discreta $X$, a qual assume diferentes valores discretos $x$,

$$
H(X)=-\sum_{i=1}^{n} p\left(x_{i}\right) * \log \left[p\left(x_{i}\right)\right]=E\left[\log \left(1 / p\left(x_{i}\right)\right]\right.
$$

onde $E[$. ] denota o operador expectação. O logaritmo do recíproco da probabilidade no argumento de $\mathrm{E}$ é a incerteza $\mathrm{H}_{\mathrm{i}}$ sobre um evento específico (um fluxo específico) do qual $p\left(X_{i}\right)$ é a probabilidade e, para repetir, $H(X)$ é uma incerteza global (para todos os fluxos). Assim, $H(X)$ decresce quando alguém tem mais informação sobre $X$. Se a variável $X$ descreve um processo determinístico, $H(X)=0$, i.e., há uma certeza absoluta de que somente um evento $X$ é possível.

A unidade na qual a entropia é medida depende da base do logaritmo utilizado na eq. (3). Se o logaritmo está na base 2, a entropia é expressa em bits. Se a base logarítmica é neperiana e(natural), então a entropia é expressa em nats.

O valor máximo de $\mathrm{H}(\mathrm{X})$ é atingido, quando a distribuição é uniforme, i.e., se nada é conhecido sobre a probabilidade $p\left(x_{i}\right)$, cada categoria é assumida como equiprovável, $p\left(x_{i}\right)=1 / n$. Nesse caso a incerteza global é máxima.

$$
H_{\max }=-\sum_{i=1}^{n}(1 / n) * \log (1 / n)=-n *(1 / n) * \log (1 / n)=\log (n)
$$

Como afirmou Ulanowicz $(1986,87)$, "a incerteza é intuitivamente maior sob máxima ignorância". Par maiores informações e interpretações sobre H(X), ver Deco, Obradovic (1997) e, também, Kapur, Kesavan (1992).

Se $H(X)$ é uma incerteza residual, após $\underline{n}$ observações, obtendo a probabilidade de várias categorias (fluxos), $\left[\mathrm{H}_{\max }-\mathrm{H}(\mathrm{X})\right]$ é a redução da incerteza resultante das $n$ observações e, assim, é a informação inerente à distribuição de $\mathrm{p}_{\mathrm{i}}$. Isso nos permite definir um importante parâmetro chamado índice de redundância:

$$
\mathrm{h}=\left(\mathrm{H}_{\max }-\mathrm{H}\right) / \mathrm{H}_{\max }=1-\mathrm{H} / \mathrm{H}_{\max }
$$

É fácil ver que como $0 \leq \mathrm{H} / \mathrm{H}_{\max } \leq 1,0 \leq \mathrm{h} \leq 1$. Esse parâmetro é muito importante porque é um índice informacional e dimensional, independente de $n$. Ele tem sido usado em várias aplicações em biologia, geografia e agroecossistemas (ver Svirezhev, Brovkon, Denisenko, 1995, 6).

É evidente que, se estamos tratando com uma estrutura complexa, precisamos explicitar a idéia de uma dimensão de capacidade. Para definir a capacidade específica ou o coeficiente de tamanho, partimos do conceito de 
Marcos Ximenes Ponte, Donald L. Van Dyne

dimensão generalizada(2) do matemático Felix Hausdorff (1919) (sobre dimensão em sistemas complexos, ver também Schroeder, 1991, Voss, 1988, e Çambel, 1993). Faremos algumas considerações sobre o sistema, para obter um parâmetro adimensional que expresse o tamanho do sistema necessário para produzir uma unidade de energia. Assim obtemos

$$
\mathrm{C}_{\mathrm{s}}=1.128 * \rho^{*} * \operatorname{LnN} / \varepsilon \mathrm{p}^{3 / 2 * \mathrm{P}_{\mathrm{r}}^{1 / 2}}
$$

onde $\rho$ é a densidade $\left[\mathrm{Kg} / \mathrm{m}^{3}\right]$ do produto agrícola, $\mathrm{N}$ é o número de fluxos do sistema, $\varepsilon_{\mathrm{p}}$ é a energia específica final produzida por unidade de produto [J/ $\mathrm{Kg}$, $\mathrm{P}_{\mathrm{r}}$ é a produção por unidade de área cultivada $\left[\mathrm{Kg} / \mathrm{m}^{2}\right]$, ou por unidade de área necessária, se quisermos levar em conta o efeito da rotação (shifting cultivation effect).

Agora, é plausível postular uma relação similar a (2), na forma

$$
\eta=\eta\left[h, c_{s^{\prime}}\right]
$$

\section{Análise dos Sistemas Agroindustriais}

A partir dessa formulação, fizemos cálculos computacionais e obtivemos os dados que se seguem. Primeiramente, a Figura 2 mostra que, a despeito da similaridade geral das estruturas dos sistemas estudados (visto que possuem os mesmos compartimentos), no nível organizacional, há grandes diferenças. É possível também notar uma forte correlação entre a eficiência e o índice de redundância. Como seria esperado, a eficiência cresce com a redundância. Isso denota que, à medida que diminui a homogeneidade da alocação de energia entre os fluxos, aumenta a diversidade, aumenta também o nível de estruturação do sistema, cresce a redundância, aumentando, também em conseqüência, a eficiência.

Figura 2 - Relação entre eficiência e índice de redundância h[-], para diferentes sistemas, para uma situação real típica.

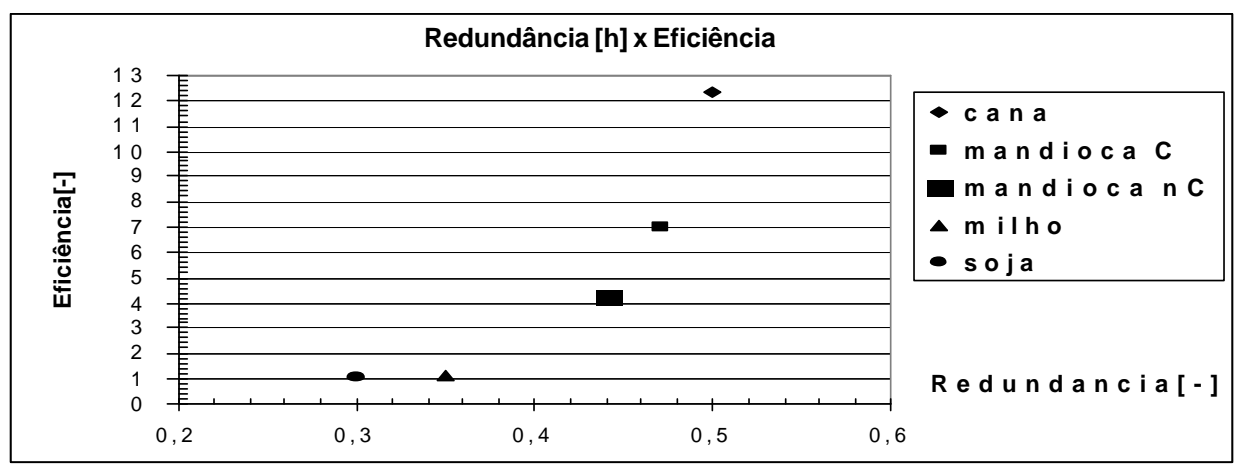

Nota: segundo dados básicos para milho e soja de Pimentel, 1980, Stout, 1990 e Shapouri, 1995; para cana Macedo e Horta, 1996; para mandioca, de Canto et alii, 1997.

\footnotetext{
Nós não usamos o exato conceito de Hausdorfft, mas somente parte da idéia de dimensão generalizada, na qual se representa o tamanho total de um objeto por [L] e, então, cobre-se ou enche-se esse espaço com $\mathrm{N}(\mathrm{r})$ pequenos cubos ou esferas de dimensão [r]. Assumimos, então, que a dimensão característica de um sistema agroindustrial poderia ser $D \mu \ln N /(1 / r)$.
}

54 
A Figura 3 mostra como a capacidade específica Cs depende do índice de redundância $h$. É importante relembrar que a capacidade específica é a forma adimensional do diâmetro em cuja área o sistema pode produzir (exportar) uma unidade de energia. Esses resultados concordam com a análise fenomenológica, baseada nas leis da termodinâmica, segundo as quais, se o sistema é mais estruturado, com um grau de hierarquia mais elevado, ele é, em conseqüência, mais produtivo (mais eficiente) e também mais compacto. Os resultados mostrados nas Figuras 2 e 3 expressam muito bem essa correlação. 0 ponto referente à soja não foi incluído na Figura 3, por ser muito alto 0 valor da capacidade específica $(C s=6.59)$. Mas isso era esperado, visto que 0 índice de redundância $h=0.303$ é muito baixo. No que diz respeito à mandioca, no sistema camponês mostrado na Figura 3, não foi considerado o efeito da itinerância, o shift effect, previsto na equação 1 . Se nós considerássemos esse efeito, o valor de Cs seria 11.278 para o mesmo valor da redundância h.

Figura 3 - Relação entre o índice de redundância e o coeficiente de tamanho, para situações reais típicas.

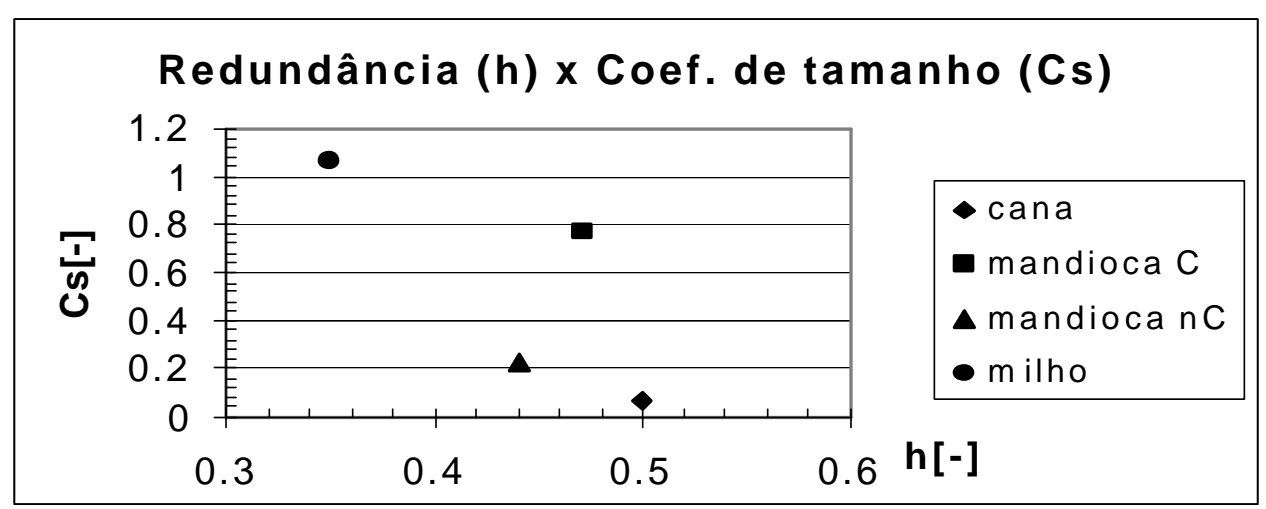

Nota: dados básicos de Macedo e Horta, 1996; Shapouri 1995; e Canto et alii, 1997.

Isso denota que o sistema camponês é mais lento, porque segue o "tempo da natureza". Ele reconstitui a fertilidade no nível agricultável do solo. Também é justificável e esperado que na Figura 3 o ponto representativo do sistema camponês esteja um pouco deslocado dos demais. Isso acontece porque o sistema camponês é estruturalmente, ou qualitativamente, diferente dos demais, isto é, pertence a outra família.

As Figuras 4, 5, 6 e 7 mostram o comportamento nos planos $\eta=\eta[h$, $\mathrm{C}_{\mathrm{s}}$ ] e o efeito do uso dos resíduos. Esses resultados mostram que a eficiência $\eta$ e a capacidade específica $C_{s}$ variam monotonicamente com o grau de organização representado pelo îndice de redundância $\mathrm{h}$.

Como estamos tratando com parâmetros adimensionais, ao analisarmos o comportamento de um sistema as variações quantitativas não deveriam alterar o campo $\mathbf{h} \times \mathbf{c}$ ou $\mathbf{h x} \mathbf{C s}$, se estas mudanças forem somente quantitativas, em nível insuficiente para produzir modificações orgânicas do siste- 
Marcos Ximenes Ponte, Donald L. Van Dyne

ma. Isso é o que podemos observar na Figura 7 a qual representa o comportamento do sistema americano de produção de etanol a partir de milho, combinando variações de produtividade agrícola de 82 e 132bu/ac (Pimentel, 1980) e a produtividade industrial de 1.875 e 2.75 galões de etanol por bushel obtida em Shapouri et all, 1995. É importante verificar que os valores adimensionais praticamente coincidem o que denota que os parâmetros utilizados são adequado para uma análise de similaridade dos sistemas. É importante notar, ainda que a aproximação torna-se maior a medida em que a ascendência (redundância) aumenta. Isto também concorda com a análise fenomenológica de que se o sistema é mais estruturado as suas características intrínsecas são mais fortes e o comportamento similar do sistema é reforçado.

Podemos também notar, ao observar as Figuras 4 a 6, que o sistema etanol a partir de cana de açúcar é mais eficiente (maior $\eta$, menor $\mathrm{C}_{\mathrm{s}}$ ) e mais desenvolvido ${ }^{(3)}$ (maior h). Isso se deve à alta capacidade da cana-de-açúcar para converter energia solar em biomassa.

Devido à alta disponibilidade de resíduo com alto potencial energético, o sistema cana-de-açúcar para produção de etanol apresenta, também, um maior potencial de melhoria.

Embora o sistema de farinha de mandioca na sua forma camponesa tenha alta eficiência, sua capacidade específica é muito alta. Quando a farinha de mandioca é produzida na sua forma não camponesa, isto é, usando energia artificial na forma de fertilizante, o nível de organização (h) e a eficiência diminuem, mas a capacidade específica melhora (decresce). Isso também é expresso na Figura 8 por meio do fator $\mathrm{g}$, que representa a razão entre a produtividade em um sistema não camponês e em um camponês. Há possibilidades de se avançar nesse tipo de análise para obter al guns indicadores de sustentabilidade, definindo-se a quantidade ideal de energia utilizada e a ponderação de possíveis ganhos, no nível organizacional. Esses ganhos poderiam ser obtidos, por exemplo, se fosse dada à capoeira ou à floresta uma função produtiva, já que teriam suas possibilidades de preservação aumentadas pelo uso de energia artificial, o que aumentaria as chances de cultivo de uma mesma área, por mais tempo (sobre essa análise especifica, ver Ponte, 1999). Esses resultados revelam ainda que, entre os casos industriais estudados, o sistema etanol de cana-de-açúcar tem nível de organização e eficiência maior que os demais, vindo em segundo lugar o sistema etanol de milho, que apresenta um melhor desempenho (maior $h$, maior eficiência $\eta$ e menor $\mathrm{C}_{\mathrm{s}}$ ) que o sistema de óleo combustível de soja.

A Tabela 1 mostra a importância relativa do trabalho e da energia artificial, revelando que os sistemas milho e soja são mais dependentes de energia artificial. 0 mais alto valor do sistema soja é produzido no estágio de conver-

\footnotetext{
É fundamental, para o tipo de análise desenvolvida neste artigo, o conceito de crescimento e desenvolvimento concebido por Ulanowicz $(1986,96)$, para uma rede de fluxos entre compartimentos. Para esse autor, o número de compartimentos e, mais importante, a quantidade global circulante no sistema caracterizam o crescimento do sistema. 0 desenvolvimento também está relacionado ao crescimento, mas há componentes do desenvolvimento que não estão relacionados ao tamanho do sistema e sim ao aumento do nível de organização, ou seja, à articulação entre os compartimentos. Esses efeitos são sistematizados em um parâmetro definido por Ulanowicz como ascendência.
}

56 
são do óleo de soja. Nesse estágio, são demandados $87.01 \%$ da energia primária requerida para o Biodiesel Life Cycle (ver USDA, USDE, 1998, 15).

Figura 4 - Etanol de cana-de-açúcar de São Paulo-Brasil (produtividade agrícola variando de 65 a 90t/ha)
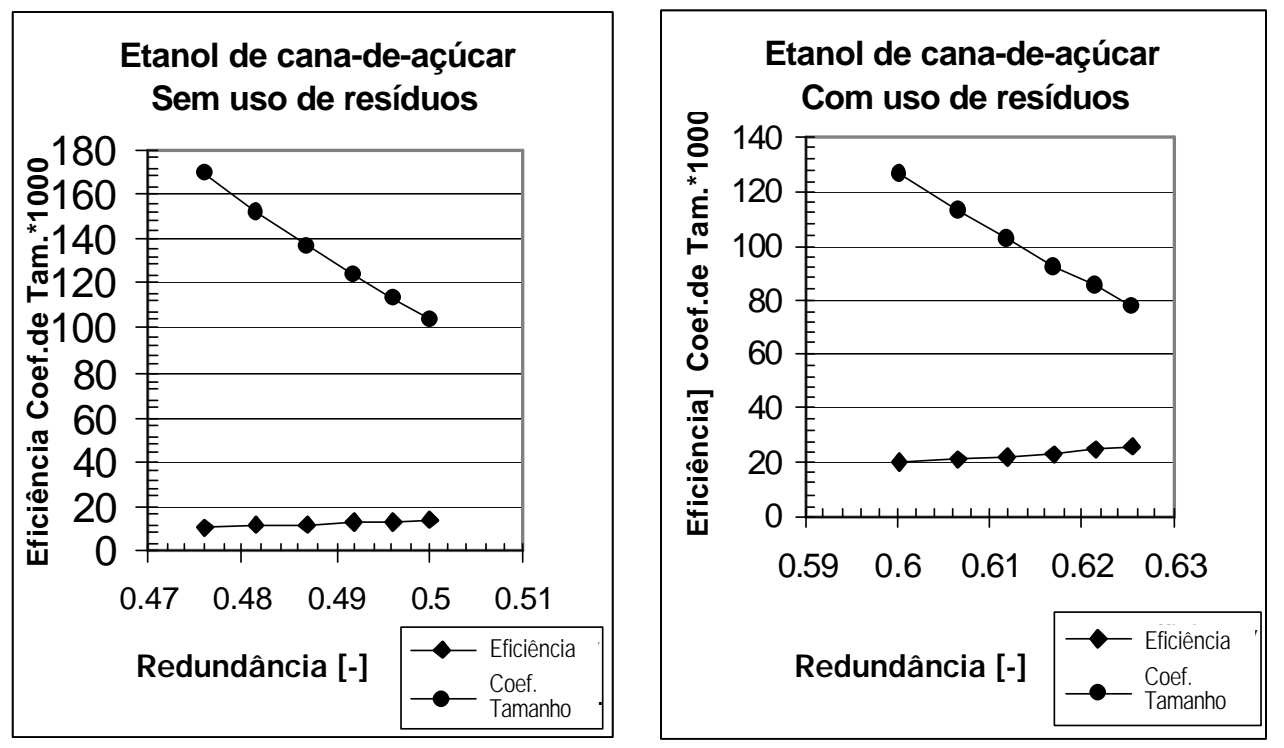

Notas: (a) Segundo Marcelo H orta, 1996, usa parte do bagaço para gerar vapor e eletricidade para uso interno; (b) usa todo o bagaço e os resíduos do campo para gerar energia. A linha superior do gráfico representa $10^{3 *}$ coeficiente de tamanho, e a inferior, a eficiência.

Figura 5 - Etanol de milho, USA (produtividade agrícola variando de 82 a $132 \mathrm{bu} / \mathrm{ac})$
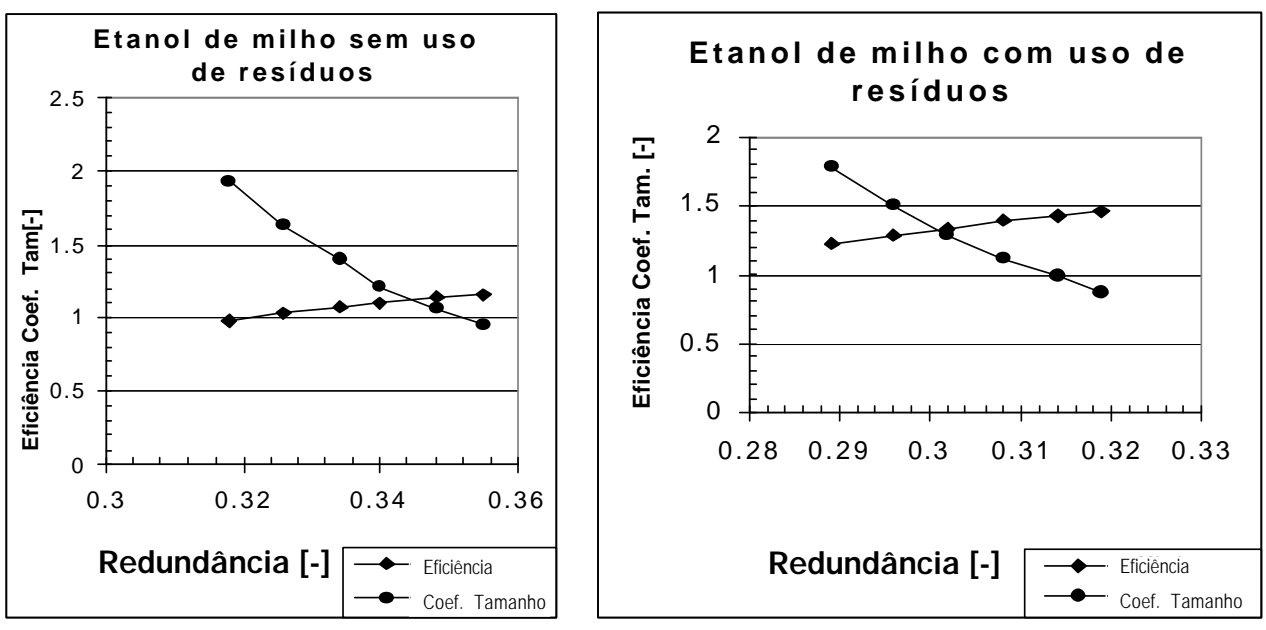

Notas: (a) Segundo Stout, 1990 e Shapouri, 1995, sem uso de resíduos agrícolas; (b) uso de $57 \%$ dos resíduos agrícolas produzidos ${ }^{(2)}$ para gerar eletricidade com $35 \%$ de eficiência. A curva superior representa o coeficiente de tamanho, e a inferior, a eficiência. 
Figura 6 - Biodiesel de soja, USA (produtividade agrícola variando de 25 a 45 bu/ac). A curva superior representa o coeficiente de tamanho, e a inferior, a eficiência.

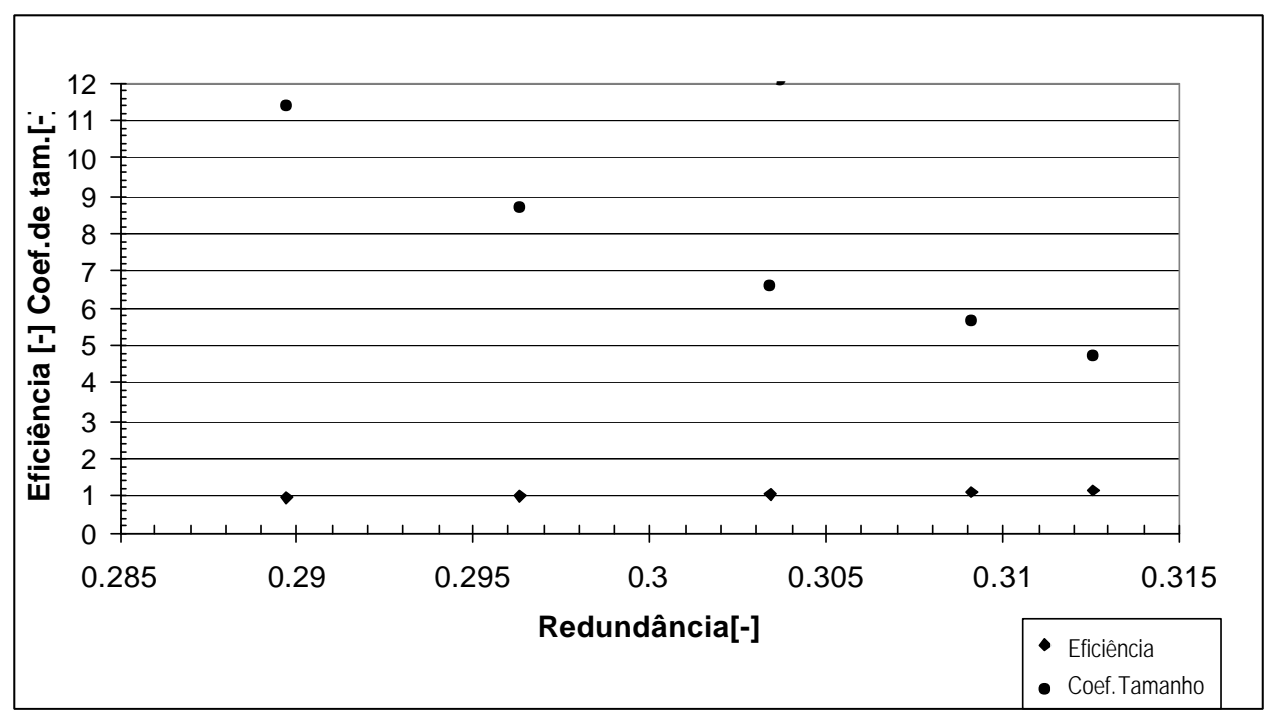

Nota: Segundo Stout, 1990 e Shapouri, 1995 (a) sem uso de resíduos agrícolas; (b) uso de $57 \%$ dos resíduos agrícolas gerados ${ }^{(2)}$ para gerar eletricidade com $35 \%$ de eficiência. A curva superior representa o coeficiente de tamanho, e o de baixo a eficiência.

Figura 7 - Etanol de milho. Várias combinações de produtividade agrícola (82 e 132bu/ac) e valores da produtividade industrial de 1.875 a 2.75 galões de etanol por bushel.

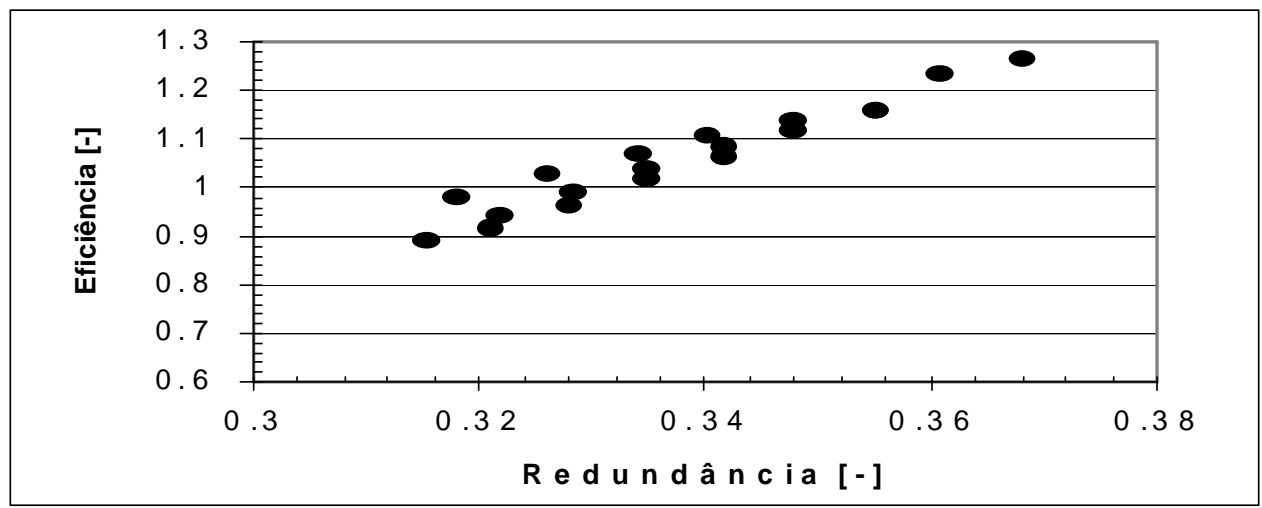

Nota: Segundo Pimentel (1980) e valores da produtividade industrial de 1.875 a 2.75 galões de etanol por bushel segundo Shapouri et alii, 1995. 
Figura 8 - Sistema de produção de farinha de mandioca.

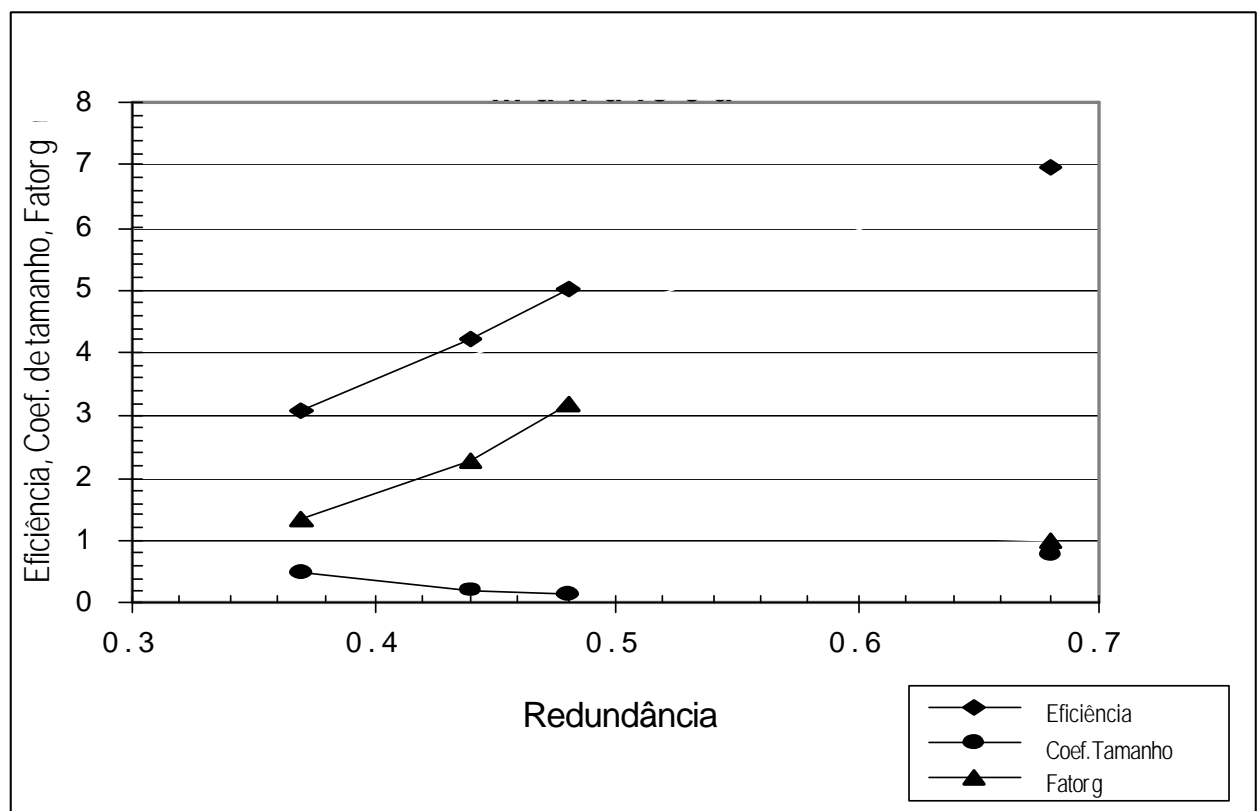

Notas: Segundo dados básicos de Canto et alii, 1997, as curvas representam o sistema não camponês. Os pontos isolados representam o sistema camponês. 0 fator $\mathrm{g}$ é a razão entre 0 valor energético dos produtos exportados(output) no sistema não camponês e no sistema camponês.

Tabela 1 - Valores dos principais parâmetros para situações típicas reais e taxas dos equivalentes energéticos do trabalho(mão-deobra) e da energia artificial utilizada em cada sistema.

\begin{tabular}{|l|c|c|c|r|r|}
\hline \multicolumn{1}{|c|}{ Parâmetros } & Unid. & $\begin{array}{c}\text { Etanol } \\
\text { de cana- } \\
\text { de- } \\
\text { açúcar }\end{array}$ & $\begin{array}{c}\text { Mandioca, } \\
\text { sistema } \\
\text { camponês }\end{array}$ & $\begin{array}{c}\text { Etanol } \\
\text { de } \\
\text { milho }\end{array}$ & $\begin{array}{c}\text { Biodiesel } \\
\text { de soja }\end{array}$ \\
\hline Redundância h & - & 0.50 & 0.47 & 0.35 & 0.3034 \\
\hline Eficiência h & - & 12.32 & 6.98 & 1.135 & 1.065 \\
\hline Coeficiente de tamanho C & - & 0.069 & 0.767 & 1.067 & 6.59 \\
\hline Mão-de-obra/energia exportada & $\%$ & 0.33 & 3.24 & 0.58 & 0.96 \\
\hline Mão-de-obra/energia importada & $\%$ & 4.08 & 22.61 & 0.66 & 1.02 \\
\hline Energia artificial/energia exportada \% & 7.47 & 0.58 & 87.41 & 92.9 \\
\hline Energia artificial/energia importada \% & 92.28 & 4.07 & 99.24 & 98.98 \\
\hline
\end{tabular}

Fonte: Canto et alii, 1997; Stout, 1990 e Shapouri, 1995; Marcelo Horta, 1996; Pimentel, 1980; Macedo e Horta, 1996. Nota: Em relação ao sistema de mandioca empresarial, cultivada com o uso de insumos modernos, detectou-se uma variabilidade muito grande na produtividade e na quantidade dos insumos, não tendo sido possível eleger uma situação típica. Inclusive verificaram-se casos, como algumas experiências de cultivos no Maranhão, em que a adubação química não trouxe nenhuma melhora na produtividade. Optou-se, assim, na obtenção dos resultados apresentados, por variar a produtividade de 15 a 35 t/ha, considerando-se situações como a de Tome Açu, com produtividade de 43t/h, exceções. 


\section{Referências bibliográficas}

BERG, C. International Molasses and Alcohol Report. 1997.

ÇAMBEL, A. B. Applied chaos theory: a paradigm for complexity. New York: Academic Press, 1993.

CONTO, A. J . et al. Sistemas de produção da farinha de mandioca no nordeste paraense. Boletim de Pesquisa da EMBRAPA (Centro de Pesquisa Agroflorestal da Amazônia Oriental), Belém, n. 97, 1997.

DECO, G.; Obradovic. An information-theoretic approach to neural computing. New York: Spring, 1996.

DENISENKO, E. A.; Svirezhev, Y. M.; Brovkin, V. A. 1991. Informational measures as method for agroecosystems analysis. J ournal of General Biology, n. 52(5), p. 691-698, 1991(em russo).

HAUSDORFF, F. Dimension und äussers mass. Mathematische Annalen, $n$. 79, p. 157-179, 1919.

JACKSON, T.; Clift, R. Where's the profit in industrial ecology? J ournal of Industrial Ecology, Cambridge, n. 2(1), p. 3-8, 1998.

KAPUR, J. N.; Kesavan, H. K. Entropy optimization principles with applications. New York: Academic Press, 1992.

MACEDO, I. C.; Horta, N. L. A. Balanço de energia na produção de cana-deaçúcar e álcool nas usinas cooperadas. Brasília, DF: Ministério de Ciência e Tecnologia, 1996.

NRC-CSAEHT. National Research Council - Committee on Sustainable Agriculture and the Environment in the Humid Tropics. Washington, D.C.: National Academy Press, 1993.

PONTE, M. X. A sustainability analysis of biomass use by information entropy theory. In: FOURTH BIOMASS CONFERENCE OF THE AMERICAS. Golden Colorado, 1999.

PONTE, M. X.; VAN DYNE, D. L. Enhanced biofuels production: development of more efficient production and product combinations. Proceedings of the $8^{\text {th }}$ Biennial Conference - Bioenergy 98, vol. II. Madson, Wisconsin, 1998.

SHANON, C. E. A mathematical theory of comunicationn. The Bell System J ournal, v. XXVII, p. 379-423 and 623-656, 1948.

SCHROEDER, M. Factrals, Chaos, Power Laws. New York: W. H. Freeman an Co., 1991.

SHAPOURI, H.; Duffield, A. J .; Graboski, M. S. 1995. Estimating the net energy balance of corn ethanol. Agricultural Economic Report, United States Department of Agriculture, n. 721, 1995.

SVIREZHEV, Y. M.; Brovkin, V. A.; Denisenko, E. A. 1995. Agroecosystem analysis approach based on the flows of artificial energy and information. Working Paper. Laxenburg-Austria. International Institute for Applied Systems Analysis, 1995. 
TIEZZI, E.; Marchettini, N.; Ulgiati, S. Integrated agro-industrial ecosystems: an assessment of the sustainability of a cogenerative approach to food, energy and chemicals production by photosynthesis. In: Constanza, R. Ecological economics: the science and management of sustainability. New York: Columbia University Press, 1991.

ULANOWICZ, R. E. Growth and development: ecosystems phenomenology. New York: Spring-Verlag, 1986.

USDA and USDE (U.S Department of Agriculture and U.S. Department of Energy). Life cycle inventory of biodiesel and petroleum diesel for use in an urban bus. Final Report, 1998.

VAN DYNE, D. L.; Kaylen, M. S.; Blase, M. G. Estimating the optimum biomass refinery size for converting lignocellulosic feedsticks into ethanol and furfural. Proceedings of the $8^{\text {th }}$ Biennial Conference - Bioenergy 98, vol. I. Madson, Winsconsin, 1998.

VOSS, R. F. 1988. Fractals in Nature: from caracterization to simulation. In: Barsnsley, M. F.; Devaney,R. L.; Mandelbrot, B. B.; Peitgen, H. O.; Saupe, D.; Voss, R. V. (eds.). Fractal Images. New York: Spring-Verlag, 1998. p. 21-70.

XIMENES, T. Cassava production, division of labor, and resources managment in Eastern Pará-Brazil. In: The Seventh International Symposium of Society and Resource Management, Culture, Environment and Society. University of Missouri - Columbia, 1998. 


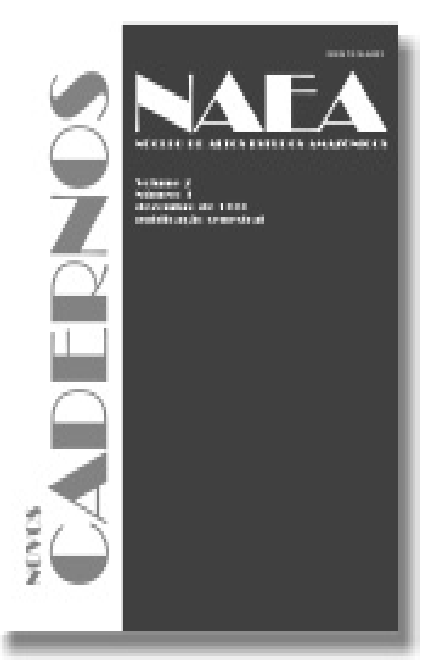

Resenhas

Dothicsfor people by thepeople, de Darnyl R.J . Marfcer

Arimar Leal Vieira

Os desbravadores: a Petrobrás e a construção do Brasil industrial, de Aldemira del Carmen A. Contreras Pearl Athur J ules Antonius

199 pp $\mathrm{R} \$ 10,00$

\section{$\mathrm{N}$ ov os Cadernos $\mathrm{N}$ aea}

V. 2 n2 (Dez 1999)

A Construção histórica do termo caboclo. Sobre estruturas erepresentações sociais no meio rural amazônico. Deborah deMagalhães Lima

A disputa pelo sangue. Reflexões sobre a constituição da identidadee "unidadetembé" SaraAlonso

Parceiros do crime: o regatão e a resistência cabocla na Amazôniatradicional.

$$
\text { DavidMcgrath }
$$

Mobilização política de comunidades negras rurais.

Domínio de um conhecimento praxiológico. RosaAcevedo Marin eEdna Ramos Castro

Unidades de conservação eas populações tradicionais. Uma análisejurídica da realidadebrasileira.

Quais fronteiras? J oséHeder Benatti

RegineSchönenberg

Do conceito defertilidadeao desustentabilidade GutembergArmando Diniz Guerra, Mariade NazaréÂngelo-Menezes

A criseecológica na agriculturafamiliar do Estado do

Maranhão: contribuição paraum debate. Antônio Carlos Reis de Freitas

Processo industrial para beneficiamento da macaxeira. Eloísa Ramos Cardoso, Sebastião Hühn,J oão de Deus B. Nascimento] únior
Menos empregos, outros trabalhos... Mudança nas políticas públicas.

Maria Cristina Cacciamali

Dimensões dainformalidadeem Belém

Ana Laura dos Santos Sena

Saúdee projetos de desenvolvimentonaAmazônia. 0 caso das hidrelétricas

RosaCarminadeSenaCouto

$236 \mathrm{pp} R \$ 10,00$

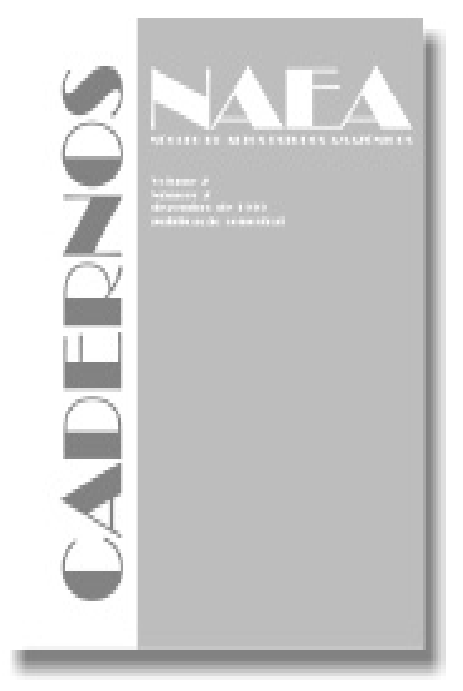

62 